\title{
ESTRUTURA DE DOIS COMPONENTES ARBÓREOS DE FLORESTA SUBTROPICAL RIPÁRIA NA CAMPANHA GAÚCHA, SANT’ANA DO LIVRAMENTO, RS
}

\author{
Ana Claudia Bentancor Araujo ${ }^{1 *}$, Helio Junior Bentancor Araujo ${ }^{2}$, Rafael Marian Callegaro ${ }^{3}$, \\ Camila Andrzejewski ${ }^{4}$, Solon Jonas Longhi ${ }^{5}$ \\ ${ }^{1 *}$ Instituto Federal de Educação Ciência e Tecnologia Farroupilha, Alegrete, Rio Grande do Sul, Brasil - ana.araujo@iffarroupilha.edu.br
${ }^{2}$ Universidade Estadual do Rio Grande do Sul, Sant'Ana do Livramento, Rio Grande do Sul, Brasil - bentancorhelinho@ gmail.com
${ }^{3}$ Universidade Federal do Espírito Santo, Departamento de Ciências Florestais e da Madeira, Jerônimo Monteiro, Espirito Santo, Brasil -
rafael.callegaro@ ufes.br
${ }^{4}$ Universidade Federal de Santa Maria, Programa de Pós-Graduação em Engenharia Florestal, Santa Maria, Rio Grande do Sul, Brasil -
camila_andrzejewski@ @otmail.com
${ }^{5}$ Universidade Federal de Santa Maria, Santa Maria, Rio Grande do Sul, Brasil - longhi.solon@gmail.com
}

Recebido para publicação: 11/08/2015 - Aceito para publicação: 05/07/2016

\begin{abstract}
Resumo
O estudo comparou a estrutura de dois fragmentos arbóreos de mata ciliar, em Sant'Ana do Livramento, RS. Para amostrar a vegetação foram instalados transectos com distância de $60 \mathrm{~m}$ entre eles, perpendiculares ao rio, de comprimento variável, conforme a largura da mata ciliar. Nesses foram instaladas parcelas contínuas de $20 \mathrm{~m}$ x $20 \mathrm{~m}$. Nas parcelas foram identificados e medidos indivíduos arbóreos vivos e mortos com diâmetro à altura do peito (DAP) igual ou maior que $5 \mathrm{~cm}$. Os índices de Shannon (H') e de Pielou (J') calculados para a Mata Ciliar com Gado (MCCG) e para a Mata Ciliar sem Gado (MCSG) foram semelhantes, $\mathrm{H}^{\prime}=2,56$ e $\mathrm{J}^{\prime}=0,76$, e $\mathrm{H}^{\prime}=2,55$ e $\mathrm{J}^{\prime}=0,74$, respectivamente. A densidade de indivíduos da MCSG (1.456,4 ind.ha ${ }^{-1}$ ) diferiu significativamente da densidade de indivíduos no MCCG (1.058,3 ind.ha $\left.{ }^{-1}\right)$. Com relação ao número de espécies, não houve diferença significativa entre as áreas. Ocotea acutifolia, Lithrea molleoides e Gymnanthes klotzschiana foram as mais representativas da MCCG e da MCSG, porém nesta a espécie L. molleoides apresentou maior densidade. A distribuição diâmetrica para o total dos fragmentos e para as espécies de maior valor de importância apresentou tendência à exponencial negativa. Palavras-chave: Fitossociologia; Pampa; gado.
\end{abstract}

\begin{abstract}
Structure of the two tree components of riparian Subtropical Forest in Campanha Gaúcha, Sant'Ana do Livramento, RS. The study compared the structure of two arboreal fragments of riparian forest in Sant'Ana do Livramento, RS. For sampling the vegetation were installed transects with a distance of $60 \mathrm{~m}$ between them, perpendicular to the river, variable length, according to the width of the riparian forest. In these plots were identified and measured individual trees living and dead showing DBH equal or above $5 \mathrm{~cm}$. The values of Shannon $\left(\mathrm{H}^{\prime}\right)$ and Pielou $\left(\mathrm{J}^{\prime}\right)$ calculated for the Riparian Forest With Cattle (RFWC) and the Riparian Forest Less Cattle (RFLC) were similar, $\mathrm{H}^{\prime}=2.56$ and $\mathrm{J}^{\prime}=0.76$, and $\mathrm{H}^{\prime}=2.55$ and $\mathrm{J}^{\prime}=0.74$, respectively. The density of individuals of RFLC (1.456,4 ind.ha $\left.^{-1}\right)$ differed significantly from the density of individuals in RFWC $\left(1058,3\right.$ ind.ha $\left.{ }^{-1}\right)$. Regarding the number of species, there was no significant difference between the areas. Ocotea acutifolia, Lithrea molleoides and Gymnanthes klotzschiana were the most representative species of the RFWC and RFLC, but that L. molleoides species is the one with higher density. The diameter distribution for the total of fragments and the species of most important value tended to the negative exponential. Keywords: Phytosociology; Pampa; cattle.
\end{abstract}

\section{INTRODUÇÃO}

O Bioma Pampa está localizado no extremo sul brasileiro, região caracterizada por uma fisionomia predominante campestre e de clima temperado, que cobre $63 \%$ do território do estado do Rio Grande do Sul (OVERBECK et al., 2007). Tal fisionomia compreende também a totalidade do território do Uruguai e parte da Argentina (SUERTEGARAY; SILVA, 2009). Dentre os domínios do bioma Pampa encontra-se a região da Campanha Gaúcha que abrange áreas levemente onduladas (relevo de coxilhas), com campos limpos (IBGE, 2006) associados às matas ciliares que acompanham as áreas úmidas, sendo essa região encontrada na porção oeste do Rio Grande do Sul. As formações florestais, nesse bioma, ocupam uma pequena área, sendo os bosques ripários as únicas distribuídas por toda a sua extensão, podendo apresentar mudanças significativas na sua composição em pequena escala espacial (KALESNICK et al., 2005).

As formações lenhosas apesar de reduzidas na região do Pampa são extensões vegetais de grande

FLORESTA, Curitiba, PR, v. 46, n. 4, p. 481 - 490, out. / dez. 2016.

Araujo, A. C. B. et al.

ISSN eletrônico 1982-4688

DOI: $10.5380 /$ rf.v46i3.42590 
importância na conservação do solo e margens de cursos d'água, contribuindo com múltiplos benefícios diretos e indiretos para os humanos, além de destinarem-se como habitat natural de uma grande quantidade de espécies animais e vegetais (GRELA; BRUSSA, 2003).

Percebe-se, no entanto, uma crescente transformação da vegetação ciliar desse bioma, áreas de preservação permanente (APP's), em cultivos agrícolas ou anteparo para o manejo pecuário. Nessa região, é comum utilizar as matas ciliares, juntamente com os campos, para o manejo do gado. No entanto, sabe-se que o acesso do gado doméstico às matas normalmente acarreta danos à estrutura da vegetação, pois, o gado alimentase de folhas, frutos e sementes palatáveis, pisoteia as plântulas e compacta o solo, dificultando a germinação das sementes e o crescimento da regeneração (WILSON, 1994). Contudo, o Brasil vive a perspectiva com o novo código florestal, Lei 12.651/2012 (BRASIL, 2012), de que áreas até então degradadas deverão ser restauradas ou recuperadas de acordo com o seu estágio de perturbação.

Nesse sentido, o presente estudo pretende verificar se é possível observar diferenças na estrutura da vegetação, em duas áreas próximas, com semelhante composição de espécies, mas que sofreram diferentes intensidades de perturbação no passado. Para isso, o estudo teve como objetivo diagnosticar e comparar a estrutura da vegetação de fragmentos de mata ciliar, com histórico de perturbação passadas e recentes, no município de Sant'Ana do Livramento, RS.

\section{MATERIAL E MÉTODOS}

O estudo foi realizado em dois fragmentos de mata ciliar no Arroio Espinilho (afluente do Rio Quaraí), localizados nas coordenadas $30^{\circ} 52^{\prime} \mathrm{S}$ e $55^{\circ} 51^{\prime} \mathrm{W}$, a uma altitude média de $208 \mathrm{~m}$, em Sant'Ana do Livramento, Rio Grande do Sul. Um dos fragmentos de mata ciliar, na década de 80, foi explorado com a retirada de espécies de interesse comercial. Após esse episódio, a área passou a ser utilizada para criação de bovinos, em torno de 0,3 unidades animal por hectare (UA/ha) durante o inverno e há dois anos o mesmo foi cercado havendo apenas um acesso para água. Essa área foi denominada, com intuito de facilitar a análise do trabalho, como Mata Ciliar sem Gado (MCSG), onde predomina uma floresta em estágio de sucessão secundária. O outro fragmento de mata ciliar, também tem um histórico de exploração semelhante à MCSG, no entanto, a prática de uso da mata ciliar como anteparo a bovinos, ovinos e equinos continua até os dias atuais, havendo livre acesso desses animais (aproximadamente 1,2 UA/ha) em toda a extensão do fragmento. Nesse local há predomínio de floresta em estágio de sucessão secundária, denominado, também para facilitação da análise do trabalho, como Mata Ciliar com Gado (MCCG). As duas áreas estão localizadas em propriedade particular e distantes $38 \mathrm{~km}$ da sede do município de Sant'Ana do Livramento, Brasil, e $40 \mathrm{Km}$ da sede do Departamento de Rivera, República Oriental do Uruguai. A pesquisa foi desenvolvida entre os anos de 2008 e 2009.

O clima da região é classificado como Cfa (Subtropical úmido, sem estação seca definida e com verões quentes), com temperatura média anual variando entre $16^{\circ} \mathrm{C} \mathrm{e} 18{ }^{\circ} \mathrm{C}$, e precipitação pluvial anual variando entre 1600 e 1900 mm (ALVARES et al., 2013). De acordo com Streck et al. (2008), toda a região da Campanha tem ampla dominância de Neossolos Litólicos ou Regolíticos Eutróficos (Unidade Pedregal). O tipo fitogeográfico da região pertence à Floresta Estacional Decidual (IBGE, 2012).

Para a análise da vegetação foram instalados transectos de comprimento variável, seis na MCSG e quatro na MCCG, sendo que os mesmos ficavam distantes $60 \mathrm{~m}$ um do outro. Para amostrar a vegetação arbórea foram distribuídas parcelas contínuas dentro dos transectos, com dimensões de $20 \mathrm{~m}$ x $20 \mathrm{~m}$. No total, foram instaladas 42 parcelas na área com gado (1,68 ha de área amostrada) e 43 parcelas na área sem gado (1,72 ha de área amostrada). Nestas foram amostrados todos os indivíduos arbóreos vivos e mortos com diâmetro à altura do peito $(\mathrm{DAP}) \geq 5 \mathrm{~cm}$. Árvores bifurcadas abaixo de 1,30 m tiveram cada fuste medido separadamente. Os dados coletados para as análises fitossociológicas das espécies foram: DAP e altura total, sendo a última medida com auxílio do hipsômetro eletrônico - Vertex III $^{\circledR}$. As espécies não identificadas foram coletadas e posteriormente identificadas no Herbário do Departamento de Ciências Florestais (HDCF) da Universidade Federal de Santa Maria (UFSM), sendo que a maioria foi identificada no campo. As espécies foram identificadas e a listagem de espécie foi organizada de acordo com o Angiosperm Phylogeny Group (APG III 2009). A confirmação e atualização dos nomes científicos deram-se por meio de consulta ao Missouri Botanical Garden (MBG) (2014).

A estrutura horizontal foi caracterizada por meio de avaliação dos descritores fitossociológicos: densidade, frequência e dominância de cada espécie, os quais foram utilizados para obter o respectivo valor de importância (VI) e determinar as espécies mais representativas da estrutura horizontal. Foram calculados os índices de diversidade de Shannon (H'), com base em logaritimo natural, e a equabilidade utilizando o índice de Pielou (J'). Para a análise da estrutura diamétrica e da estrutura vertical, seguiu-se o procedimento de Spiegel, descrito por Felfili e Rezende (2003), utilizando o DAP e a altura total, respectivamente. A partir deste procedimento, as árvores foram distribuídas em 12 classes de diâmetro, com 5,2 cm de intervalo de classe, e em 12 classes de altura, com 1,1 m de intervalo de classe. Conforme Schneider e Finger (2000) realizou-se o ajuste das frequências em classes diamétricas com a equação de Meyer e foi calculado o Quociente de Liocourt para o 
componente arbóreo. Para os cálculos foram consideradas as variáveis, número de indivíduos e de espécies. Nos dois fragmentos, foram consideradas como unidade amostral cada parcela de $400 \mathrm{~m}^{2}$. O teste t, de Student, foi aplicado nos dois grupos, para as variáveis: número de indivíduos/ha e número de espécies, com o objetivo de testar a existência de igualdade entre as médias, a 5\% de probabilidade. Para as duas variáveis analisadas, os dados foram testados quanto à sua normalidade e após esta análise aplicou-se a raiz quadrada para normalizar a variável referente ao número de espécies.

\section{RESULTADOS}

Na mata ciliar com gado (MCCG), o número de indivíduos arbóreos encontrados foi 1.778 indivíduos, com estimativa de 1.058,3 ind.ha ${ }^{-1}$ e na mata ciliar sem gado (MCSG), foram observados 2.505 indivíduos, com estimativa de $1.456,4$ ind.ha ${ }^{-1}$. A MCSG apresentou área basal de 25,2913 $\mathrm{m}^{2}$.ha ${ }^{-1}$, enquanto a área basal da MCCG foi de $28,0064 \mathrm{~m}^{2} . \mathrm{ha}^{-1}$.

Na MCCG foram encontradas 28 espécies distribuídas em 15 famílias e 30 espécies divididas em 16 famílias na MCSG (Tabela 1).

Tabela 1. Espécies arbóreas amostradas em duas matas ciliares, em Sant'Ana do Livramento, RS. MCCG Mata Ciliar Com Gado; MCSG - Mata Ciliar Sem Gado. DA - Densidade Absoluta; DR\% Densidade Relativa; FR\% - Frequência Relativa; DoR\% - Dominância Relativa; VI - Valor de Importância.

Table 1. Tree species sampled in the riparian forest, in Sant'Ana do Livramento, RS. RFWC - Riparian Forest With Cattle, RFLC - Riparian Forest Less Cattle. DA - Absolute Density; DR\% - Relative Density; FR\% - Frequency Relative; DoR\% - Relative Dominance; VI - Importance of Value.

\begin{tabular}{|c|c|c|c|c|c|c|c|c|c|c|}
\hline \multirow{2}{*}{ Família/Espécie } & \multicolumn{5}{|c|}{ MCCG } & \multicolumn{5}{|c|}{ MCSG } \\
\hline & DA & $\mathrm{DR} \%$ & FR\% & DoR\% & VI & DA & $\mathrm{DR} \%$ & FR\% & DoR\% & VI \\
\hline \multicolumn{11}{|l|}{ ANACARDIACEAE } \\
\hline Lithrea brasiliensis Marchand & 4,2 & 0,4 & 0,9 & 0,7 & 2,3 & 14,5 & 1 & 1,7 & 0,9 & 3,6 \\
\hline Lithrea molleoides (Vell.) Engl. & 188,7 & 17,8 & 9,8 & 25,8 & 53,4 & 415,7 & 28,5 & 9,2 & 22,2 & 60 \\
\hline Schinus lentiscifolius Marchand & 44,6 & 4,2 & 5,8 & 2,1 & 12,1 & 6,4 & 0,4 & 2,3 & 0,1 & 2,8 \\
\hline Schinus molle L. & 3,6 & 0,3 & 1,2 & 0,9 & 2,4 & 12,8 & 0,8 & 1,9 & 0,6 & 3,4 \\
\hline $\begin{array}{l}\text { Schinus polygamus (Cav.) } \\
\text { Cabrera }\end{array}$ & 4,2 & 0,4 & 1,2 & 0,2 & 1,7 & 4,1 & 0,3 & 1,7 & 0,1 & 2,2 \\
\hline \multicolumn{11}{|l|}{ ASTERACEAE } \\
\hline $\begin{array}{l}\text { Moquiniastrum polymorphum } \\
\text { (Less.) G.Sancho }\end{array}$ & 1,2 & 0,1 & 0,6 & 0,1 & 0,8 & 7,6 & 0,5 & 1,1 & 0,4 & 2,2 \\
\hline \multicolumn{11}{|l|}{ EUPHORBIACEAE } \\
\hline $\begin{array}{l}\text { Gymnanthes klotzschiana } \\
\text { Müll.Arg. }\end{array}$ & 118,5 & 11,2 & 7,8 & 7,1 & 26,1 & 111,6 & 7,7 & 6,7 & 7,7 & 22 \\
\hline $\begin{array}{l}\text { Gymnanthes schottiana } \\
\text { Müll.Arg. }\end{array}$ & & & & & & 1,7 & 0,1 & 0,3 & 0,04 & 0,5 \\
\hline Manihot grahamii Hook. & 1,2 & 0,1 & 0,3 & 0,1 & 0,5 & 0,6 & 0,0 & 0,3 & 0,0 & 0,3 \\
\hline Sebastiania brasiliensis Spreng. & 46,4 & 4,4 & 3,2 & 2 & 9,5 & 25,6 & 1,8 & 1,4 & 1,6 & 4,7 \\
\hline \multicolumn{11}{|l|}{ LAURACEAE } \\
\hline Ocotea acutifolia (Nees) Mez & 203,6 & 19,2 & 9,8 & 36 & 65 & 188,9 & 13 & 7 & 21,3 & 41,3 \\
\hline \multicolumn{11}{|l|}{ MYRTACEAE } \\
\hline $\begin{array}{l}\text { Blepharocalyx salicifolius } \\
\text { (Kunth) O.Berg. }\end{array}$ & 29,2 & 2,8 & 5,8 & 1,3 & 9,8 & 33,7 & 2,3 & 5,3 & 1,2 & 8,8 \\
\hline Eugenia mansoi O.Berg. & & & & & & 3,5 & 0,2 & 0,3 & 0,1 & 0,7 \\
\hline Eugenia uniflora $\mathrm{L}$. & 41,1 & 3,9 & 5,5 & 1,1 & 10,4 & 61 & 4,2 & 6,4 & 1,5 & 12,1 \\
\hline $\begin{array}{l}\text { Myrcianthes cisplatensis } \\
\text { (Cambess.) O.Berg }\end{array}$ & 53 & 5 & 7,2 & 2,4 & 14,6 & 39,5 & 2,7 & 3,4 & 1,3 & 7,4 \\
\hline $\begin{array}{l}\text { Myrcianthes pungens (O.Berg) } \\
\text { D.Legrand }\end{array}$ & 3 & 0,3 & 1,2 & 0,1 & 1,8 & 20,3 & 1,4 & 2,8 & 1,7 & 5,9 \\
\hline $\begin{array}{l}\text { Myrrhinium atropurpureum } \\
\text { Schott }\end{array}$ & 1,8 & 0,2 & 0,9 & 0,0 & 1,1 & 4,1 & 0,3 & 0,8 & 0,1 & 1,3 \\
\hline \multicolumn{11}{|l|}{ PRIMULACEAE } \\
\hline Myrsine umbellata Mart. & & & & & & 4,7 & 0,3 & 1,4 & 0,2 & 1,9 \\
\hline \multicolumn{11}{|l|}{ QUILLAJACEAE } \\
\hline $\begin{array}{l}\text { Quillaja brasiliensis (A.St.-Hil. } \\
\text { \& Tul.) Mart. }\end{array}$ & 1,8 & 0,2 & 0,9 & 0,7 & 1,7 & 21,5 & 1,5 & 2,2 & 3 & 6,7 \\
\hline
\end{tabular}

FLORESTA, Curitiba, PR, v. 46, n. 4, p. 481 - 490, out. / dez. 2016.

Araujo, A. C. B. et al.

ISSN eletrônico 1982-4688

DOI: $10.5380 /$ rf.v46i3.42590 


\begin{tabular}{|c|c|c|c|c|c|c|c|c|c|c|}
\hline \multicolumn{11}{|l|}{ RHAMNACEAE } \\
\hline Scutia buxifolia Reissek & 59,5 & 5,6 & 7,5 & 2,2 & 15,3 & 72,7 & 5 & 7,6 & 3 & 15,5 \\
\hline \multicolumn{11}{|l|}{ ROSACEAE } \\
\hline Prunus myrtifolia (L.) Urb. & 5,9 & 0,6 & 1,4 & 0,4 & 2,4 & 4,1 & 0,3 & 0,6 & 0,7 & 1,5 \\
\hline \multicolumn{11}{|l|}{ RUTACEAE } \\
\hline Zanthoxylum rhoifolium Lam. & 7,1 & 0,7 & 1,7 & 0,2 & 2,8 & 1,7 & 0,1 & 0,6 & 0,1 & 0,8 \\
\hline \multicolumn{11}{|l|}{ SALICACEAE } \\
\hline $\begin{array}{l}\text { Xylosma tweediana (Clos) } \\
\text { Eichler }\end{array}$ & & & & & & 4,1 & 0,3 & 1,4 & 0,1 & 1,8 \\
\hline \multicolumn{11}{|l|}{ SANTALACEAE } \\
\hline $\begin{array}{l}\text { Acanthosyris spinescens (Mart. } \\
\text { \& Eichler) Griseb. }\end{array}$ & 2,4 & 0,2 & 0,6 & 0,6 & 1,8 & 7 & 0,5 & 0,8 & 1,7 & 3,0 \\
\hline $\begin{array}{l}\text { Jodina rhombifolia (Hook. \& } \\
\text { Arn.) Reissek }\end{array}$ & 1,2 & 0,1 & 0,6 & 0,7 & 0,3 & & & & & \\
\hline \multicolumn{11}{|l|}{ SAPINDACEAE } \\
\hline $\begin{array}{l}\text { Allophylus edulis (A.St.-Hil. et } \\
\text { al.) Hieron. ex Niederl. }\end{array}$ & 42,8 & 4,1 & 5,2 & 3,2 & 12,4 & 43 & 3 & 5,6 & 2,2 & 10,7 \\
\hline $\begin{array}{l}\text { Allophylus guaraniticus (A.St.- } \\
\text { Hil.) Radlk. }\end{array}$ & 10,7 & 1 & 2 & 0,5 & 3,5 & 10,5 & 0,7 & 1,1 & 0,5 & 2,3 \\
\hline Matayba elaeagnoides Radlk. & 1,2 & 0,1 & 0,6 & 0,1 & 0,7 & 47,7 & 3,3 & 5,9 & 9,2 & 18,4 \\
\hline \multicolumn{11}{|l|}{ SAPOTACEAE } \\
\hline $\begin{array}{l}\text { Pouteria salicifolia (Spreng.) } \\
\text { Radlk. }\end{array}$ & 81,5 & 7,7 & 4,3 & 4,5 & 16,5 & 122,1 & 8,4 & 5,3 & 6,6 & 20,3 \\
\hline \multicolumn{11}{|l|}{ STYRACEAE } \\
\hline Styrax leprosus Hook. \& Arn. & 41,6 & 3,9 & 6,3 & 2,3 & 12,6 & 102,3 & 7 & 7,6 & 4,3 & 18,9 \\
\hline \multicolumn{11}{|l|}{ ULMACEAE } \\
\hline Celtis iguanaea (Jacq.) Sarg. & 2,4 & 0,2 & 0,3 & 0,1 & 0,6 & 4,1 & 0,3 & 0,6 & 0,1 & 0,9 \\
\hline \multicolumn{11}{|l|}{ VERBENACEAE } \\
\hline $\begin{array}{l}\text { Aloysia gratissima (Gillies \& } \\
\text { Hook.) Tronc. }\end{array}$ & 1,2 & 0,1 & 0,3 & 0,0 & 0,4 & & & & & \\
\hline Mortas & 54,7 & 5,2 & 7,5 & 4,9 & 17,5 & 59,3 & 4,1 & 6,7 & 7,3 & 18,1 \\
\hline TOTAL & 1058,3 & 100 & 100 & 100 & 300 & 1456,4 & 100 & 100 & 100 & 300 \\
\hline
\end{tabular}

Os valores de diversidade do Índice de Shannon $\left(\mathrm{H}^{\prime}\right)$ e do Índice de Pielou (J') calculados para a MCCG e para a MCSG foram: $\mathrm{H}^{\prime}=2,56 / \mathrm{J}^{\prime}=0,76$ e $\mathrm{H}^{\prime}=2,55 / \mathrm{J} '=0,74$, respectivamente.

Conforme o teste de $t$, aplicado ao nível de $5 \%$ de probabilidade, a média da densidade de indivíduos da MCSG (1.456,4 ind.ha $\left.{ }^{-1}\right)$ diferiu da MCCG (1.058,3 ind.ha $\left.{ }^{-1}\right)(t=1,66 ; \mathrm{p}<0,001)$. A riqueza média de espécies da MCSG (17,4 espécies/ha) foi superior à riqueza média de espécies da MCCG (16,6 espécies/ha), contudo, não se verificou - pelo teste $t$ - diferença significativa ( $\mathrm{p}<0,921)$.

As espécies de maior densidade na MCCG foram a Ocotea acutifolia com 203,6 ind.ha ${ }^{-1}$ (densidade relativa $(\mathrm{DR})=19,2 \%)$, Lithrea molleoides com 188,7 ind.ha $^{-1}(\mathrm{DR}=17,8 \%)$, Gymnanthes klotzschiana com 118,5 ind.ha $^{-1}(\mathrm{DR}=11,2 \%)$ e Pouteria salicifolia com 81,5 ind.ha ${ }^{-1}(\mathrm{DR}=7,7 \%)$. O. acutifolia e L. molleoides obtiveram a maior frequência relativa, ambas com o mesmo valor (frequência relativa (FR) $=9,8 \%$ ). O. acutifolia apresentou a maior dominância absoluta $\left(6,2 \mathrm{~m}^{2} \cdot \mathrm{ha}^{-1}\right)$, perfazendo uma dominância relativa de $36 \%$ e o maior valor de importância $(65 \%)$, sendo seguida em ordem decrescente de valor de importância pela $L$. molleoides $(53,4 \%)$ e $G$. klotzschiana $(26,1 \%)$ as quais, juntas, contribuíram com 48,2\% do valor total (300\%) do VI. As árvores mortas obtiveram o quarto melhor valor de importância $(17,5 \%)$, com consideráveis valores de frequência, dominância e densidade (Tabela 1).

$\mathrm{Na}$ MCSG, ao contrário da MCCG, Lithrea molleoides foi a espécie de maior densidade com 415,7 ind.ha $^{-1}(\mathrm{DR}=28,5 \%)$, seguida por Ocotea acutifolia com 188,9 ind.ha $^{-1}(\mathrm{DR}=13 \%)$, Pouteria salicifolia com 122,1 ind.ha ${ }^{-1}(\mathrm{DR}=8,4 \%)$, Gymnanthes klotzschiana com 111,6 ind.ha ${ }^{-1}(\mathrm{DR}=7,7 \%)$ e Styrax leprosus com 102,3 ind.ha $^{-1}(\mathrm{DR}=7 \%)$. L. molleoides obteve a maior frequência relativa $(\mathrm{FR}=9,2 \%)$, decorrente de uma frequência absoluta de 76,7\%. A maior dominância absoluta foi apresentada pela espécie L. molleoides com $3,265 \mathrm{~m}^{2} \cdot \mathrm{ha}^{-1}$, perfazendo uma dominância relativa de $22,2 \%$ e o maior valor de importância $(60 \%)$, sendo seguida em ordem decrescente de valor de importância pela O. acutifolia $(41,3 \%)$ e G. klotzschiana $(22 \%)$ as quais, juntas, contribuem com $41,1 \%$ do valor total $(300 \%)$ do VI. Nesse fragmento as árvores mortas também apresentaram um alto valor de importância $(18,1 \%)$ (Tabela 1).

Tanto a MCCG quanto a MCSG apresentaram maior concentração de árvores nas classes de diâmetros inferiores (Figura 1Aa) (Figura 1Ba). 

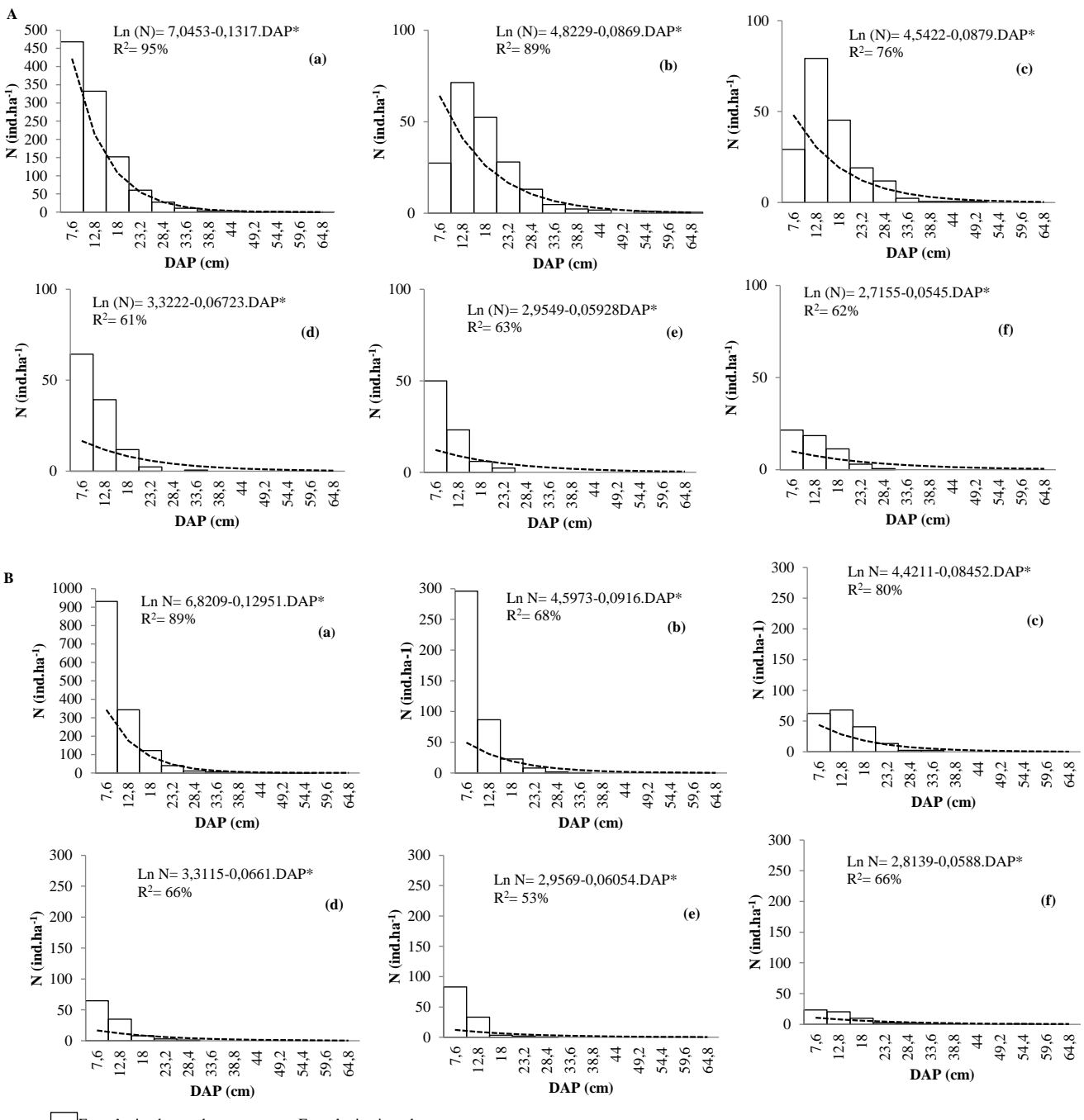

Figura 1. MCCG (A): distribuição de frequências observadas e ajustadas nos centros de classes de diâmetros do componente arbóreo (Figura 1Aa) das espécies Ocotea acutifolia (Figura 1Ab), Lithrea molleoides (Figura 1Ac), Gymnanthes klotzschiana (Figura 1Ad) e Pouteria salicifolia (1Ae), e árvores mortas (Figura 1Af) e MCSG (B): distribuição de frequências observadas e ajustadas nos centros de classes de diâmetros do componente arbóreo (Figura 1Ba) das espécies Lithrea molleoides (Figura 1Bb), Ocotea acutifolia (Figura 1Bc), Gymnanthes klotzschiana (Figura 1Bd) e Pouteria salicifolia (Figura 1Be), e árvores mortas (Figura 1Bf). Santana do Livramento, RS. 2010. * DAP = diâmetro à altura do peito.

Figure 1. RFWC (A): distribution of observed frequencies and adjusted in centre of each diameter classes of the tree component (Figure 2Aa) species Ocotea acutifolia (Figure 2Ab), Lithrea molleoides (Figure 2Ac), Gymnanthes klotzschiana (Figure 2Ad) and Pouteria salicifolia (1Ae), and dead trees (Figure 2Af) and RFLC (B): distribution of observed frequencies and adjusted in centre of each diameter classes of the tree component (Figure 2Ba) species Lithrea molleoides (Figure 2Bb), Ocotea acutifolia (Figure 2Bc), Gymnanthes klotzschiana (Figure 2Bd) and Pouteria salicifolia (Figure 2Be), and dead trees (Figure 2Bf). Sant'Ana do Livramento, RS. 2010.

A curva de frequência ajustada pela equação de Meyer evidencia que a estrutura diamétrica da mata ciliar tanto no fragmento com gado quanto no fragmento sem gado não se encontra balanceada, com déficit de indivíduos nas classes diamétricas com pontos médios iguais a 33,6; 38,8; 44 e 49,2 cm (MCCG) (Figura 1Aa), e nos pontos médios 23,2; 28,4; 33,6; 38,8 e $44 \mathrm{~cm}$ (MCSG) (Figura 1Ba). O valor estimado para o Quociente de Liocourt (q) na MCCG foi de 1,14 e para a MCSG foi de 0,83.

A análise da estrutura diamétrica das espécies de maior valor de importância na MCCG revela que apenas Gymnanthes klotzschiana (Figura 1Ad) e Pouteria salicifolia (Figura 1Ae) apresentaram tendência a exponencial negativa. As outras espécies, Ocotea acutifolia (Figura 1Ab) e Lithrea molleoides (Figura 1Ac)

FLORESTA, Curitiba, PR, v. 46, n. 4, p. 481 - 490, out. / dez. 2016.

Araujo, A. C. B. et al.

ISSN eletrônico 1982-4688

DOI: $10.5380 /$ rf.v46i3.42590 
apresentaram um baixo número de indivíduos na menor classe de diâmetro, entre 5,0 e 10,2 cm. Padrão oposto ao observado para a estrutura diamétrica das espécies de maior VI na MCSG, onde L. molleoides (Figura 1Bb), G. klotzschiana (Figura 1Bd) e Pouteria salicifolia (Figura 1Be) exibiram tendência a exponencial negativa. Apenas Ocotea acutifolia (Figura 1Bc) apresenta um pequeno déficit de indivíduos na menor classe de diâmetro entre 5,0 e $9,0 \mathrm{~cm}$.

Os indivíduos mortos na MCCG apresentaram o quarto maior VI e sua amplitude foi de 5 a $31 \mathrm{~cm}$ de diâmetro. A maior densidade (41,07 ind.ha $\left.{ }^{-1}\right)$ ficou restrita as duas menores classes de diâmetro $5 \mathrm{~cm} \leq \mathrm{DAP}<$ 10,2 cm / 10,2 cm $\leq$ DAP $<15,4 \mathrm{~cm}$ (Figura 1Af). Na MCSG a amplitude dos indivíduos variou entre 5 e 41,4 cm de diâmetro, e a maior densidade (43,6 ind.ha $\left.{ }^{-1}\right)$ ficou limitada as duas menores classes de diâmetros $5 \mathrm{~cm} \leq$ DAP $<10,2 \mathrm{~cm} / 10,2 \mathrm{~cm} \leq$ DAP $<15,4 \mathrm{~cm}$ (Figura 1Bf)

Ao comparar os limites de diâmetros das árvores mortas $(5,0 \mathrm{~cm} \leq \mathrm{DAP}<31 \mathrm{~cm})$ com o componente arbóreo $(5,0 \mathrm{~cm} \leq \mathrm{DAP}<67,4 \mathrm{~cm})$ da MCCG verificou-se a ausência de árvores mortas com diâmetros grandes, no entanto, é possível verificar a existência, ainda que pequena, de árvores mortas com diâmetro médio.

Na MCCG 59,7\% das árvores estão agrupadas em três classes intermediárias de altura, com valores centrais entre 4,7 e 6,9 m (Figura 2Aa). Na MCSG observaram-se duas classes intermediárias de agrupamento, com valores centrais de 5,8 e 6,9 m (Figura 2Ba).
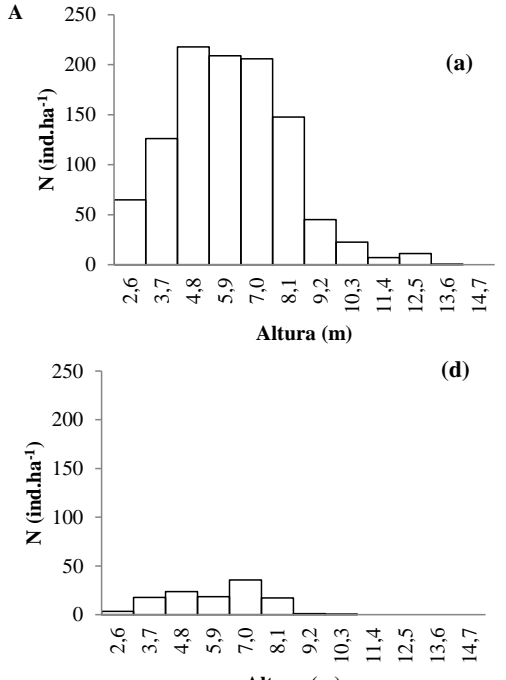

B
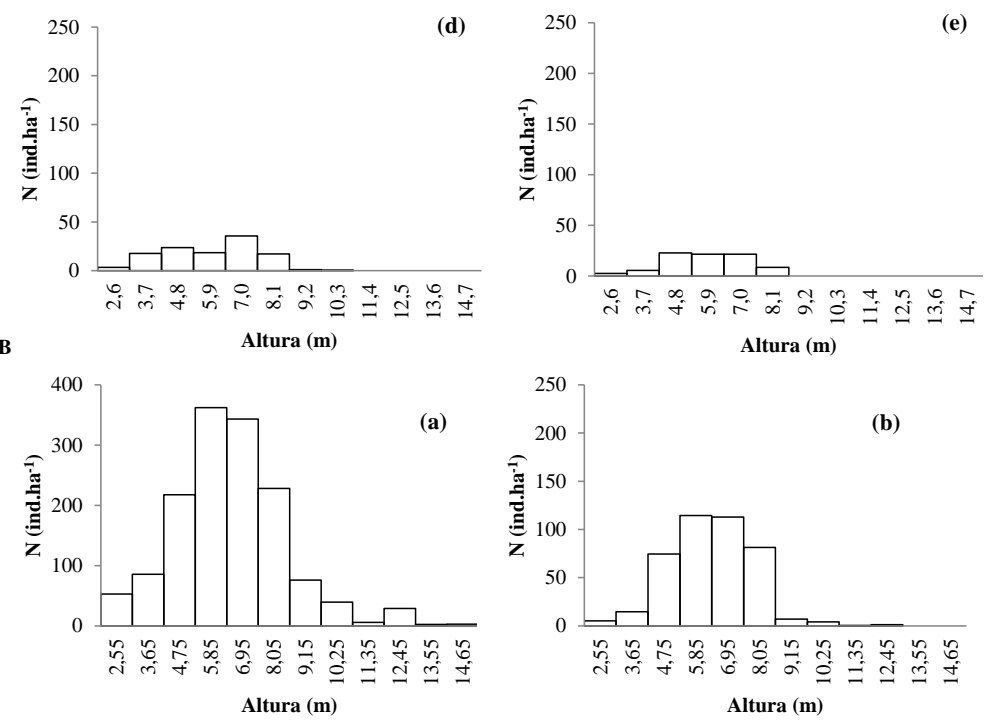

(d)

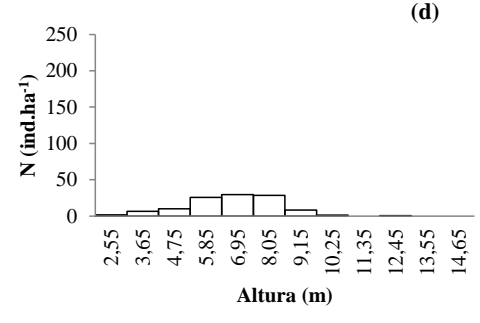

(e)
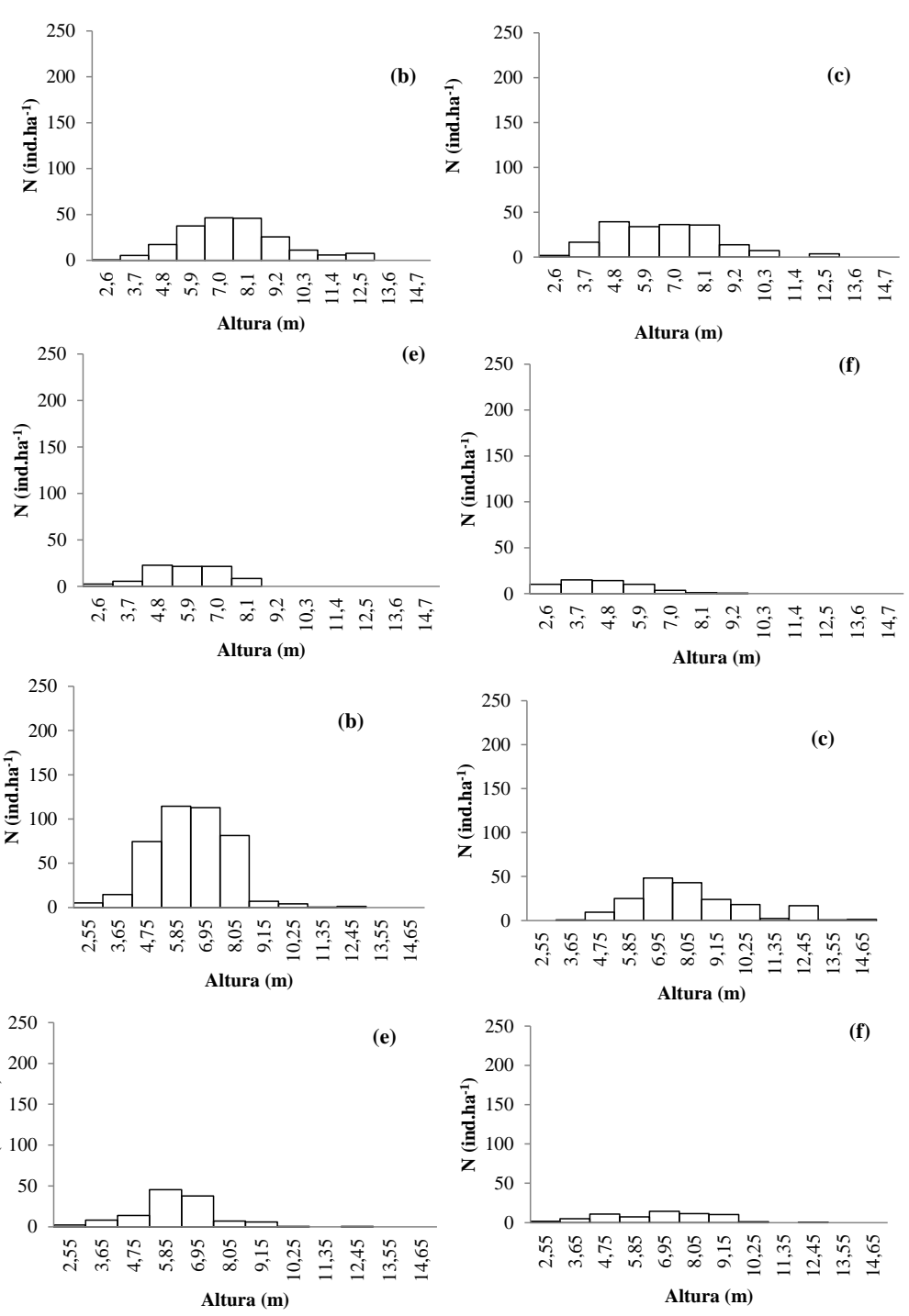

Figura 2. MCCG A: estrutura vertical do componente arbóreo (Figura 2Aa) das espécies Ocotea acutifolia (Figura 2Ab), Lithrea molleoides (Figura 2Ac), Gymnanthes klotzschiana (Figura 2Ad) e Pouteria salicifolia (Figura 2Ae), e árvores mortas (Figura 2Af) e MCSG B: estrutura vertical do componente arbóreo (Figura 2Ba) das espécies Lithrea molleoides (Figura 2Bb), Ocotea acutifolia (Figura 2Bc), 
Gymnanthes klotzschiana (Figura 2Bd) e Pouteria salicifolia (Figura 2Be), e árvores mortas (Figura 2Bf). Sant'Ana do Livramento, RS. 2010.

Figure 2. RFWC A: vertical structure of the tree component (Figure 2Aa) species Ocotea acutifolia (Figure 2Ab), Lithrea molleoides (Figure 2Ac), Gymnanthes klotzschiana (Figure 2Ad) and Pouteria salicifolia (Figure 2Ae), and dead trees (Figure 2Af) and RFLC B: vertical structure of the tree component (Figure 2Ba) species Lithrea molleoides (Figure 2Bb), Ocotea acutifolia (Figure 2Bc), Gymnanthes klotzschiana (Figure 2Bd) and Pouteria salicifolia (Figure 2Be), and dead trees (Figure 2Bf). Sant'Ana do Livramento, RS. 2010.

Ao avaliar a distribuição de altura das populações de espécies com maior VI da MCCG verificou-se nas mesmas, a tendência à distribuição normal (Figura 2Ab, 2Ac, 2Ad e 2Ae). Padrão semelhante de estrutura vertical é observado na MCSG onde é possível verificar que as populações de $O$. acutifolia (Figura $2 \mathrm{Bb}$ ) e $L$. molleoides (Figura $2 \mathrm{Bc}$ ) apresentam sua distribuição em altura muito semelhante ao componente arbóreo. $\mathrm{O}$ contrário foi observado para as espécies Gymnanthes klotzschiana (Figura 2Bd) e Pouteria salicifolia (Figura 2Be) que apresentaram um padrão diferenciado do dossel florestal.

A concentração de árvores mortas na MCCG restringiu-se ao estrato inferior, enquanto que na MCSG as árvores mortas concentraram-se em indivíduos do estrato inferior e médio.

\section{DISCUSSÃO}

A MCSG apresentou densidade de indivíduos maior que a MCCG, no entanto, a área basal observada na MCSG é menor que na MCCG, sugerindo a concentração de indivíduos de menor diâmetro na área sem gado. Grela (2003) observou padrão semelhante ao estudar dois fragmentos de mata ciliar no rio Lunarejo (Departamento de Rivera/UY) em que o fragmento de mata ciliar com histórico de degradação apresentou 4.364 indivíduos/ha e $30,25 \mathrm{~m}^{2} / \mathrm{ha}$ de área basal, enquanto que o fragmento de mata ciliar sem nenhum tipo degradação antrópica apresentou 2.036 indivíduos/ha e 50,06 m²/há. Conforme o autor a área de mata ciliar com histórico de perturbação encontra-se em estágio secundário de regeneração com muitos indivíduos, porém estes de menor tamanho resultando em uma área basal menor. Os valores de riqueza de espécies botânicas encontrados para a MCSG e para a MCCG são semelhantes aos observados por Grela (2003) que encontrou no fragmento com histórico de degradação 29 espécies e no fragmento sem degradação 30 espécies.

Observa-se que os valores de diversidade encontrados na mata ciliar sem gado e na mata ciliar com gado são semelhantes, indicando que as duas áreas apresentam condições ambientais similares, devido, em parte, à proximidade dos dois fragmentos. Os valores de diversidade de Shannon e de Pielou obtidos para o MCCG e para a MCSG são semelhantes aos valores encontrados por Kilca et al. (2012), em duas florestas ribeirinhas do rio Piratini, RS, onde o autor obteve valores de $\mathrm{H}^{\prime}=2,85 / 2,51$ e J' $=0,79 / 0,69$, e superiores aos valores obtidos por De Marchi e Jarenkow (2008) em um fragmento de floresta ribeirinha no rio Camaquã, onde os pesquisadores encontraram $H^{\prime}=2,34$ e J'=0,69. Quanto ao Índice de Pielou é possível presumir que existe certa uniformidade na distribuição dos indivíduos entre as espécies amostradas em ambos os fragmentos estudados, já que este índice mede a proporção da diversidade observada em relação à máxima diversidade esperada (KANIESKI et al., 2010).

Possivelmente o melhor estado de conservação da MCSG, média de densidade de indivíduos e de número de espécies superior a MCCG, esteja relacionado ao histórico de utilização da área, pois, foi informado pelo proprietário que em anos anteriores, antes do isolamento da mesma, o número de animais dentro desse fragmento não era alto e até mesmo, em algumas épocas, a área permaneceu sem animais.

Constatou-se que $O$. acutifolia e $L$. molleoides apresentaram altos valores de densidade, dominância e frequência tanto na mata ciliar com gado quanto na mata ciliar sem gado. De acordo com Sobral et al. (2006), $O$. acutifolia é comum na região da Campanha Gaúcha, principalmente em áreas úmidas e, L. molleoides é uma espécie pioneira que ocorre tanto em locais secos quanto úmidos, sendo comum encontrá-la, principalmente, em formações secundárias.

Piaggio e Delfino (2009) estudando as matas ciliares do arroio de Corrales, no Departamento de Rivera, encontraram Gymnanthes klotzschiana e Pouteria salicifolia dentre as espécies de maior valor de importância para a área de estudo. Os pesquisadores ressaltaram que a presença de $P$. salicifolia na mata ciliar estudada ocorria preferencialmente em banhados onde a porcentagem de umidade no solo era maior, isso porque se trata de uma espécie hidrófila.

Tanto a MCCG quanto a MCSG apresentaram distribuição dos indivíduos nas classes diamétricas com tendência à exponencial negativa, com concentração de indivíduos nas classes inferiores De acordo com Machado et al. (2010), a distribuição diamétrica é um reflexo das características autoecológicas das espécies, ou seja, não é o fato de possuir maior densidade que as espécies irão garantir sua manutenção na comunidade, mas sim sua

FLORESTA, Curitiba, PR, v. 46, n. 4, p. 481 - 490, out. / dez. 2016.

Araujo, A. C. B. et al.

ISSN eletrônico 1982-4688

DOI: $10.5380 /$ rf.v46i3.42590 
capacidade de competir dentro do seu nicho ecológico (SCHAAF et al., 2006). Portanto, embora o número maior de indivíduos nas classes menores sugira que as espécies estão garantidas na estrutura futura da floresta, há necessidade de análises mais detalhadas para garantir interpretações mais seguras das distribuições diamétricas.

Pode-se observar que na MCCG, 95,6\% dos indivíduos amostrados restringem-se ao intervalo de DAP entre 5,0 e 20,6 cm, enquanto que na MCSG, 95,8\% dos indivíduos amostrados limitam-se ao intervalo de DAP entre 5,0 e 15,4 cm. O elevado valor de indivíduos $(63,9 \%)$ de menor diâmetro $(5,0-10,2 \mathrm{~cm})$ na mata ciliar sem gado pode ser resultado da menor influência do pisoteio e pastejo do gado no passado para a área e, possivelmente o contrário tenha ocorrido na mata ciliar com gado. Para Machado et al. (2004), a grande quantidade de indivíduos pequenos e finos pode indicar a ocorrência de severas perturbações no passado, o que corrobora com as intervenções antrópicas ocorridas anteriormente nas áreas avaliadas.

A curva de frequência ajustada de Meyer evidenciou que a estrutura diamétrica, tanto da MCSG quanto da MCCG, não se encontra balanceada. Conforme Machado et al. (2004), fatores inerentes à fragmentação, como forma e tamanho do fragmento florestal, dificultam o recrutamento de indivíduos de algumas espécies, o que, consequentemente, contribui para a ocorrência de uma estrutura não balanceada. Callegaro et al. (2012) observaram padrão semelhante para uma Floresta Estacional Decidual ripária em Jaguari, RS, e atribuíram a estrutura não balanceada do fragmento a fase intermediária de desenvolvimento sucessional em que o mesmo se encontrava. Tal fato sugere que, no futuro, as espécies pioneiras tenderiam a ser substituídas, equilibrando a estrutura diamétrica.

Os valores estimados para o Quociente de Liocurt para a MCCG $(1,14)$ e para a MCSG $(0,83)$ são inferiores ao valor encontrado por Callegaro et al. (2012), onde o mesmo encontrou q=1,69 para Floresta Estacional Decidual ripária. Outra observação para o Quociente de Liocourt nas duas áreas avaliadas, é que os mesmos não se comportaram de forma constante, variando tanto na mata ciliar com gado quanto na mata ciliar sem gado nas diferentes classes de diâmetro.

A análise da estrutura diamétrica das espécies de maior valor de importância na MCCG revela que apenas Gymnanthes klotzschiana e Pouteria salicifolia apresentaram tendência a exponencial negativa. As outras espécies, Ocotea acutifolia e Lithrea molleoides apresentaram um baixo número de indivíduos na menor classe de diâmetro, entre 5,0 e 10,2 cm. Padrão oposto ao observado para a estrutura diamétrica das espécies de maior VI na MCSG onde L. molleoides, G. Klotzschiana e $P$. salicifolia exibem tendência a exponencial negativa. Apenas $O$. acutifolia apresenta um pequeno déficit de indivíduos na menor classe de diâmetro entre 5,0 e $9,0 \mathrm{~cm}$.

Quando as espécies apresentam um baixo número de indivíduos nas classes menores há a possibilidade destas estarem enfrentando problemas de recrutamento, o que resulta em dificuldade de formar um expressivo banco de imaturos no sub-bosque (MACHADO et al., 2010). A partir das curvas de frequências diamétricas ajustadas para as espécies de $O$. acutifolia e L. molleoides na MCCG, foi detectado o desequilíbrio entre as taxas de mortalidade e recrutamento de indivíduos destas espécies, evidenciado pela discrepância entre as frequências observadas e ajustadas. Conforme Paula et al. (2004), quando isso ocorre, pode-se inferir que o ciclo de vida das espécies não está se completando, indicando que estas espécies estão sendo paulatinamente substituídas por outras de fases mais avançadas da sucessão florestal.

A ausência de árvores mortas com diâmetros grandes, tanto na MCCG quanto na MCSG, sugerem que sob a influência de árvores dominantes os indivíduos menores acabam por sucumbir em razão dos inúmeros fatores de competição.

De maneira geral, as duas matas podem ser consideradas como de baixa altura, com poucos indivíduos na maior classe de altura $(14,1 \mathrm{~m}-15,2 \mathrm{~m})$. Possivelmente, tais resultados estão relacionados às limitações impostas pelo seu meio físico, pois, as duas comunidades arbóreo crescem em solo raso e rochoso o que provavelmente limita o desenvolvimento das espécies. Resultados semelhantes, para mata ciliar, foram obtidos por De Marchi e Jarenkow (2008), que encontraram o maior número de indivíduos entre as classes de altura de $6 \mathrm{~m}$ a $11 \mathrm{~m}(85,17 \%)$. Assim como no presente estudo, estes, também consideraram a floresta com formação de dossel descontínuo.

\section{CONCLUSÕES}

- A análise das estruturas diamétricas nos dois locais sugere que os mesmos possuem boa capacidade de renovação, entretanto, não se encontram balanceadas, mostrando que existe possibilidade de mudança nas suas estruturas.

- A estrutura vertical permite deduzir que as duas matas ciliares tendem a concentrar maior número de árvores no dossel intermediário.

- O objetivo do estudo foi parcialmente atingido, pois, apesar de existirem diferenças, ainda que sutis, entre as áreas avaliadas nesse estudo, ressalta-se que para se chegar a conclusões a respeito dos efeitos que o gado 
exerce sobre a estrutura das florestas adultas ripárias e suas espécies na Campanha Gaúcha há necessidade de um período maior e contínuo de estudo.

\section{REFERÊNCIAS}

ALVARES, C. A.; STAPE, J. L.; SENTELHAS, P. C.; GONCALVES, J. L. de M.; SPAROVEK, G. Köppen's climate classification map for Brazil. Meteorologische Zeitschrift, Stuttgart, v. 22, n. 6, p. 711-728, 2014.

ANGIOSPERM PHYLOGENY GROUP - APG III. An update of the Angiosperm Phylogeny Group classification for the orders and families of flowering plants - APG III. Botanical Journal of the Linnean Society, London, v. 161, p. 105-121, 2009.

BRASIL. Lei $\mathrm{n}^{\circ}$ 12.651, de 25 de maio de 2012. Dispõe sobre a proteção da vegetação nativa; altera as Leis $\mathrm{n}^{\text {os }}$ 6.938, de 31 de agosto de 1981, 9.393, de 19 de dezembro de 1996, e 11.428, de 22 de dezembro de 2006; revoga as Leis $\mathrm{n}^{\text {os }} 4.771$, de 15 de setembro de 1965, e 7.754, de 14 de abril de 1989, e a Medida Provisória $\mathrm{n}^{\circ}$ 2.166-67, de 24 de agosto de 2001; e dá outras providências. Diário Oficial da União, Brasília, DF, vinte cinco de maio de 2012. Disponível em: 〈http://www.planalto.gov.br/ccivil_03/_ato2011-2014/2012/lei/112651.htm> Acesso em: 16 Mar. 2015.

CAlleGARO, R. M.; LONGHI, S. J.; ARAUJO, A. C. B.; KANIESKI, M. R.; FLOSS, P. A.; GRACIOLI, C. R. Estrutura do componente arbóreo de uma floresta estacional decidual ripária em Jaguari, RS. Ciência Rural, Santa Maria, v. 42, n. 2, p. 305-311, 2012.

DE MARCHI, T. C.; JARENKOW, J. A. Estrutura do componente arbóreo de mata ribeirinha no rio Camaquã, município de Cristal, Rio Grande do Sul, Brasil. Iheringia, Série Botânica, Porto Alegre, v. 63, n. 2, p. 241248, 2008.

FELFILI, J. M.; REZENDE, R. P. Conceitos e métodos em fitossociologia. Brasília: Universidade de Brasília, 2003. 68 p.

GRELA, I. A. Evaluácion Del estado sucesional de um bosque subtropical de Quebrada em El norte de Uruguay. Acta Botanica Brasilica, São Paulo, v. 17, n. 2, p. 315-324, 2003.

GRELA, I; BRUSSA, C. Relevamiento florístico y análisis comparativo de comunidades arbóreas de Sierra de Ríos (Cerro Largo - Uruguay). Revista Agrociencia, Pelotas, v. 7, n. 2, p. 11-26, 2003.

INSTITUTO BRASILEIRO DE GEOGRAFIA E ESTATÍSTICA (IBGE). Manual Técnico da Vegetação Brasileira. Rio de Janeiro: IBGE. 2012. 271 p.

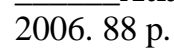

Atlas das representações literárias de regiões brasileiras - Brasil Meridional. Rio de Janeiro: IBGE,

KALESNICK, K. F.; CAGNONI, P.; BERTOLINI, P.; QUINTANA, R.; MARAMES, N.; MALVÁREZ, A. La vegetación del refugio educativo de la Ribera Norte, Província de Buenos Aires, Argentina. Invasión de espécies exóticas. Insugeo, Tucumán, v. 14, p. 139-150, 2005.

KANIESKI, M. R.; ARAUJO, A. C. B.; LONGHI, S. J. Quantificação da diversidade em Floresta Ombrófila Mista por meio de diferentes Índices Alfa. Revista Scientia Forestalis, Piracicaba, v. 38, n. 88, p. 567-577, 2010.

KILCA, R. V.; SOARES, J. C. W.; SOUZA, A. M.; MEDEIROS, E. M. de; JARENKOW, J. A. Cambios florísticos y estructurales entre dos comunidades arbóreas de um bosque ripário bajo condiciones ambientales contrastantes en la Pampa sur brasileña. Iheringia, Série Botânica, Porto Alegre, v. 67, n. 2, p. 165-175, 2012.

MARCHIORI, J. N. C. Fitogeografia do Rio Grande do Sul: campos sulinos. Porto Alegre: EST, 2004. 110 p.

MISSOURI BOTANICAL GARDEN (MBG). Explore the beta release of web TROPICOS. Disponível em: <http://www.tropicos.org/> Acesso em: 22 de Out. de 2014.

MACHADO, E. L. M.; GONZAGA, A. P. D.; CARVALHO, W. A. C.; SOUZA, J. S.; HIGUCHI, P.; SANTOS, R. M. dos; SILVA, A. C. da; OLIVEIRA-FILHO, A. T. de. Flutuações temporais nos padrões de distribuição diamétrica da comunidade arbóreo-arbustivo e de 15 populações em um fragmento florestal. Revista Árvore, Viçosa, v. 34, n. 4, p. 723-732. 2010.

MACHADO, E. L. M.; OLIVEIRA-FILHO, A. T. de; CARVALHO, W. A. C.; SOUZA, J. S.; BORÉM, R. A. T.; BOTEZELLI, L. Análise comparativa da estrutura e flora do compartimento arbóreo-arbustivo de um remanescente florestal na fazenda Beira Lago, Lavras, MG. Revista Árvore, Viçosa, v. 28, n. 4, p. 499-516, 2004. 
OVERBECK, G.; MÜlleR, S. C.; FIDELIS, A.; PFADENHAUER, J.; PILlAR, V. de P.; BLANCO, C. C.; BOLDRINI, I. I.; BOTH, R.; FORNECK, E. D. Brazil's neglected biome: The South Brazilian Campos. Perspectives in Plant Ecology, Evolution and Systematics, Germany, v. 9, p. 101-116, 2007.

PAUlA, A. de.; DA SIlVA, A. F.; JUNIOR, P. de M.; DOS SANTOS, F. A. M; DE SOUZA, A. L. Sucessão ecológica da vegetação arbórea em uma Floresta Semidecidual, Viçosa, MG, Brasil. Acta Botanica Brasilica, São Paulo v. 18, n. 3, p. 407-423, 2004.

PIAGGIO, M.; DELFINO, L. Florística y fitosociología de un bosque fluvial en Minas de Corrales, Rivera, Uruguay. Ilheringa, Serie Botânica, Porto Alegre, v. 64, n. 1, p. 45-51, 2009.

SOBRAL, M.; JARENKOW, J. A. (Orgs.). Flora arbórea e arborescente do Rio Grande do Sul, Brasil. São Carlos: RiMa: Novo Ambiente, 2006. 350 p.

SCHNEIDER, P. R.; FINGER, C. A. G. Manejo sustentado de florestas inequiânias heterogêneas. Santa Maria: UFSM, 2000. 195 p.

SUERTEGARAY, D. M. A.; SILVA, L. A. P. Tchê Pampa: histórias da natureza gaúcha. In: PILLAR, V. P.; MÜlleR, S. C.; CASTILHOS, Z. M. S.; JACQUES, A. V. A. (Eds.). Campos Sulinos: conservação e uso sustentável da biodiversidade. Brasília: Ministério do Meio Ambiente, 2009. p. 42-62.

SCHAAF, L. B.; FILHO, A. F.; GALVÃO, F.; SANQUETA, C. R. Alteração na estrutura diamétrica de uma Floresta Ombrófila Mista no período entre 1979 e 2000. Revista Árvore, Viçosa, v. 30, n. 2, p. 283-295, 2006.

STRECK, E. V.; KAMPF, N.; DALMOLIN, R. S. D.; KLAMT, E.; NASCIMENTO, P. C. do; SCHNEIDER, E.; PINTO, L. F. S. Solos do Rio Grande do Sul. 2. ed. Porto Alegre: EMATER/RS, 2008. 222 p.

WILSON, H. D. Regeneration of native forest on Hinewai reserve, Banks peninsula. New Zealand Journal of Botany, New Zealand, v. 32, n. 3, p. 373-383, 1994. 